\title{
Interactive Image Repair with Assisted Structure and Texture Completion
}

\author{
Teryl Arnold \\ blossom1625@yahoo.com \\ Bryan S. Morse \\ morse@byu.edu
}

Follow this and additional works at: https://scholarsarchive.byu.edu/facpub

Part of the Computer Sciences Commons

\section{Original Publication Citation}

T. Arnold and B. S. Morse, "Interactive image repair with assisted structure and texture completion," in WACV '7: Proceedings of the Eighth IEEE Workshop on Applications of Computer Vision, IEEE Computer Society, February 27.

\section{BYU ScholarsArchive Citation}

Arnold, Teryl and Morse, Bryan S., "Interactive Image Repair with Assisted Structure and Texture Completion" (2007). Faculty Publications. 273.

https://scholarsarchive.byu.edu/facpub/273 


\title{
Interactive Image Repair with Assisted Structure and Texture Completion
}

\author{
Teryl Arnold and Bryan S. Morse \\ Department of Computer Science \\ Brigham Young University \\ 3361 TMCB, Provo, UT 84602 \\ morse@cs.byu.edu
}

\begin{abstract}
Removing image defects in an undetectable manner has been studied for its many useful and varied applications. In many cases the desired result may be ambiguous from the image data alone and needs to be guided by a user's knowledge of the intended result. This paper presents a framework for interactively incorporating user guidance into the filling-in process, more effectively using user input to fill in damaged regions in an image. This framework contains five main steps: first, the scratch or defect is detected; second, the edges outside the defect are detected; third, curves are fit to the detected edges; fourth, the structure is completed across the damaged region; and finally, texture synthesis constrained by the previously computed curves is used to fill in the intensities in the damaged region. Scratch detection, structure completion, and texture synthesis are influenced or guided by user input when given. Results include removal of defects from images that contain structure, texture, or both structure and texture. Users can complete images with ambiguous structure in multiple ways by gesturing the cursor in the direction of the desired structure completion.
\end{abstract}

\section{Introduction}

Removing unwanted scratches or objects from an image in an undetectable manner has been studied for its many useful and varied applications. Approaches to this problem go by names such as inpainting, image completion, filling in, or image repairing. A number of these approaches also have their roots in the related problem of texture synthesis.

This paper presents a method for detecting local structure, completing that structure through the damaged region, and synthesizing texture in the region by drawing from source regions that are topologically consistent with the local structure. Specifically, we contribute an interactive framework through which the user can influence the direction of the structural completion and resolve ambiguities.

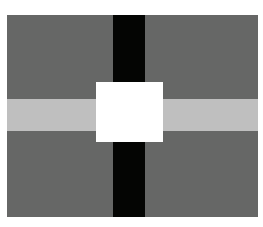

(a) Ambiguous Case

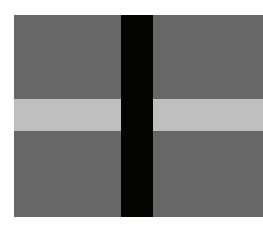

(b) Vertical Gesture

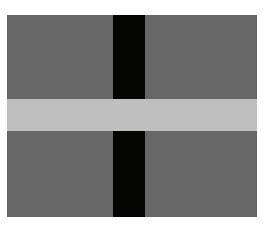

(c) Horizontal Gesture
Figure 1. User-guided image repair in an ambiguous case. With simple mouse gestures during the completion process, the user specifies both a directional preference for structural completion and a priority for filling (b: vertical, c: horizontal).

\subsection{Related Work}

Many inpainting techniques propagate the greyscale structure of the surrounding region into the damaged region in order to smoothly reconstruct the level curves $[2,3,4,5$, $7,17,21]$. These methods usually work well for removing small, thin scratches or areas in an image, but they tend to introduce blurring for large areas. While often able to repair smooth structure, they are unable to reproduce texture.

Other approaches fill in the target region by synthesizing texture from the surrounding area $[1,8,12,13,15,22,23]$. These are very successful when the surrounding area is a single texture but often fail when the damage spans multiple textured regions. While texture-synthesis methods are good at reproducing smaller-scale structure within textures, they are not as good at recovering larger-scale changes in texture.

Bertalmio et al. [6] attempt to simultaneously recover both structure and texture by separating the greyscale structure from the texture. They then repair the greyscale structure through PDE-based inpainting, repair the texture through texture synthesis, and recombine the results. Because the two processes are independent, the texture synthesis is not constrained by the structure, sometimes causing slight misalignment of greyscale and texture boundaries. 


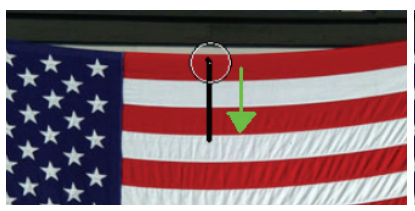

(a) Initial mouse click

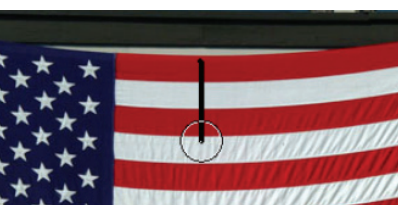

(b) User drags mouse

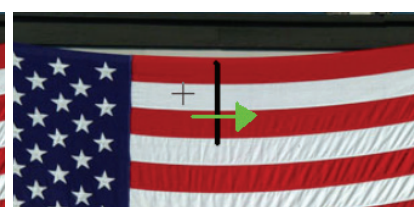

(c) Gesture to guide completion

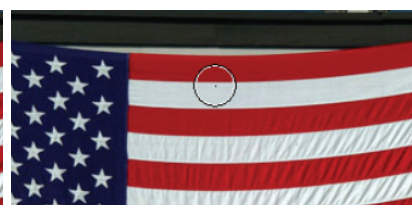

(d) Final click to stop

Figure 2. User interaction process. The user right-clicks the mouse in the defect (a) and drags the cursor across the areas to consider in identifying both the damaged and valid image regions (b). If necessary, the user left-clicks the mouse and drags in the direction of desired structure completion (c). In this case, the user indicates completion of the flag's stripes using a horizontal motion. Another right-click ends the repair (d). Green arrows represent the direction of the cursor motion.

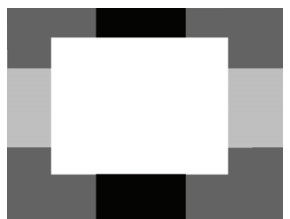

(a) Original Image

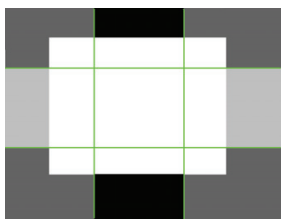

(e) Completion (2nd Phase)

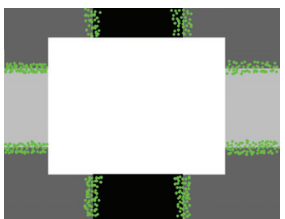

(b) Scattered Edge Points

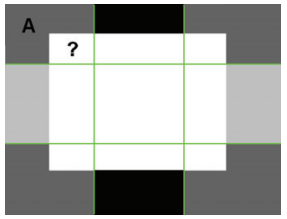

(f) Constrained Search

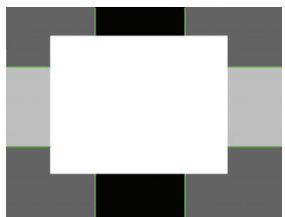

(c) Edge Fitting

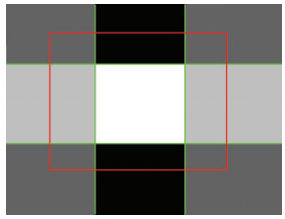

(g) Unambiguous Fill-in

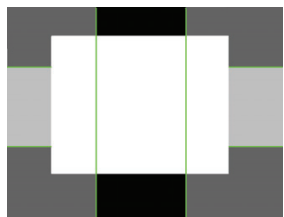

(d) Completion (1st Phase)

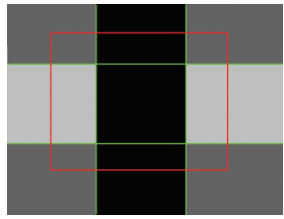

(h) Priority Filling

Figure 3. The repair process. After the user clicks in the damaged region (a, magnification of Figure 1a) and drags the cursor over both the damaged and relevant "good" areas, a simple regiongrowing algorithm separates the damage from the valid image. Edge detection then finds structure in the surrounding area (b). These sparse and perhaps noisy edge points are then fitted with rotated polynomials (c), which are connected using an optimization process guided by optional user gestures (d). A second phase connects the remaining structure (e). Texture synthesis with a constrained search fills in the damaged region with appropriate textures $(f, g)$. Some cases are ambiguous (overconstrained), so a priority-based filling process is used, again using information from the user's gestures (h, vertical gesture).

Drori et al. [11] and Demanet et al. [10] attempt similar approaches, but rather than diffusing greyscale structure they diffuse segmentation maps that determine from which source region synthesized textures are drawn.

Criminisi et al. [9] also try to incorporate structure using a priority-based texture synthesis. This method first synthesizes texture along directions propagated from exterior structural edges. It then synthesizes the remainder of the texture in non-structural regions.

Sun, et al. [20] take this further and first introduce user interaction into the repair by allowing the user to first draw curves for the important structures passing through the damaged region. These curves guide a priority-based filling whereby texture blocks along these curves are first generated and optimized through belief propagation. These curves then also serve to limit the source regions for the search much like in the earlier work in [10,11,14].

Jia and Tang [14] attempt to automatically determine the structural edges in the surrounding area and connect them through the damaged region. Unlike [20], this connection occurs automatically through a voting process than rather requiring user-drawn curves. These connected curves then limit the search process for texture synthesis.

One problem frequently cited in this work is the problem of depth ambiguity $[2,11,20$, among others] as shown in Figure 1. Some methods allow limited resolution of ambiguities through pre-specified preferences (vertical vs. horizontal, brighter over darker, favoring symmetry, etc.) [11] or manual layering [20], but none provide for direct user input to resolve ambiguities during the filling-in process. 


\subsection{Overview and User Interaction}

Using an interactive algorithm that leverages user gestures to fill in damaged regions, cases such as Figure 1 can be completed according to the user's intent. We implement this in a framework similar to that of Jia and Tang [14], but unlike their method user gestures influence the completion of the structure into the damaged region. Unlike the approach of Sun, et al. [20], the method requires only simple directional gestures rather than explicitly drawing each curve of the missing structure.

User input influences the process in four ways:

1. The user indicates which region to remove and what surrounding data to consider in the filling-in process (Figure 2a,b).

2. Optional directional gestures guide completion of the underlying structure in the damaged region (Figure 2c).

3. User gestures also determine priority of which edges to consider when valid source areas cannot be uniquely determined in relation to the detected structure.

4. The user decides when the result is satisfactory by continuously viewing new results produced by the algorithm and indicating when stop (Figure 2d). At any point they may make additional directional gestures to guide the repair.

\section{Framework and Methods}

Our framework for interactive image repair includes identifying the damaged and valid regions, detecting the surrounding structure, fitting curves to that structure, completing the structure interrupted by the damaged region, and filling in the damaged area using texture synthesis constrained by the surrounding and completed structure.

\subsection{Identifying Regions}

The user indicates the region to repair by right-clicking the mouse within the damaged region. They then drag the cursor to identify other regions to consider. The area the user drags the cursor over is segmented to be either part of the defect or part of the valid surrounding data using a region-growing algorithm seeded by the user's initial mouse click. Although more sophisticated segmentation methods could easily be incorporated (most inpainting or texture synthesis methods simply assume that the area to repair has already been manually identified), we have found this to be suitable for many applications and provides "one-click" repair rather than separate actions for identification then repair. The user does not need to keep the mouse positioned within the defect as they paint it. The initial mouse click seeds the region-growing process, and the user need only pass the area of the cursor over all of the desired defect.

All areas the user paints with the cursor that are not part of the region-grown defect are considered valid source data for the texture synthesis process. This allows the user to specify the valid source regions without requiring a separate step. We have also found this to be faster than searching the entire image for matches [13] and more effective than simply searching a fixed-size area around the defect.

\subsection{Edge Structure Detection and Fitting}

As with [14], we detect structure surrounding the defect and complete this missing structure through the region.

\subsubsection{Structure Detection}

Before finding edges, we first apply anisotropic diffusion [18] to reduce noise while preserving the edges.

Since the surrounding image may have finer-grained texture, we detect edges by comparing the color characteristics over larger regions around the potential edge point. The average intensity for each color channel is computed in four separate $3 \times 3$ quadrants surrounding the pixel. These are then combined to give color features as in [16] and reduced to a single weighted sum (feature) for each quadrant.

Once the average color features are computed across the four neighboring regions, the differences between the regions are computed. Absolute differences are calculated horizontally, vertically, and diagonally. The maximum difference computed for all pixels is used to determine a difference threshold. Maximum differences above that threshold are considered edge pixels. Figure $3 b$ shows the edges detected in the example image shown in Figure 3a. After the edge pixels are detected in the valid data, they are grouped spatially to represent separate edges.

\subsubsection{Structure Fitting}

After edge detection, each edge is represented as a group of (potentially scattered) pixels as shown in Figure 3b. To accurately complete structure, the location of pixels relative to these edges must be known during the texture synthesis process, so we fit these scattered edge points with curves (Figure 3c). For efficiency in determining relationships between these curves and other pixels, we use low-degree rotated polynomials [19].

Sets of edge pixels can be represented by rotated polynomials by first computing the orientation of each set of edge points. For each edge set, the points are then translated so that their mean is at the origin and rotated so that their orientation aligns with the $x$-axis. A least-squares fit is then used to approximate the points by a low-degree polynomial, and the mean, orientation, and polynomial coefficients are 
stored for the curve. (In the illustrative example in Figure $3 \mathrm{~d}$ the curves are just lines, but that need not be the case.)

\subsection{Structure Completion}

Some of the detected structure could be interrupted by the damaged region. As a result, some of the previously computed curves should be combined to form the correct structure across the damaged region. For the case illustrated in Figure 3, the white square in the middle is the region to remove, so those eight edges are the only ones to be considered in the structure completion process. Without the square there, it is apparent that the top two edges should connect respectively to the bottom two while the two edges on the left should connect respectively to the two edges on the right. A combination of user input and the geometry of the fitted curves can be used to successfully combine the computed eight edges into four so that the underlying structure extends across the damaged region successfully (Figure 3d,e).

More specifically, there are two main steps for connecting the underlying structure. The first step is a search that is executed only if user input is given. If user input is not given, the algorithm continues straight to the second step. The second step executes a similar search to attempt to match up any curves that have not yet been paired.

\subsubsection{First Search (Given User Input)}

The first search uses the following metrics to determine whether curves should be combined:

1. mean squared error for the combined sets of edge points if the curves were combined (MSE),

2. angular difference between the user motion and the direction between the two curves' endpoints $\left(\operatorname{diff}_{\theta}\right)$, and

3 . difference between colors adjacent to the edges.

The first search completes structure by minimizing the weighted sum of these metrics subject to MSE $<T_{1}$ and $\operatorname{diff}_{\theta}<T_{2}$, where the thresholds $T_{1}$ and $T_{2}$ specify maximum values allowed for a specific criterion. Figure $3 \mathrm{~d}$ shows the result after the first search is implemented on the image and structures shown in Figure $3 \mathrm{c}$ when the user strokes the cursor in a vertical direction.

\subsubsection{Second Search (Remaining Edges)}

The second search then uses the following metrics to determine whether the remaining curves should be combined:

1. mean squared error for the combined sets of edge points if the curves were combined (MSE),

2. difference between colors adjacent to the edges,

3. Euclidean distance between the endpoints of the curves,

\section{4. difference between curve orientations.}

The second search completes structure by minimizing the weighted sum of these metrics subject to MSE $<T_{1}$, where the threshold $T_{1}$ is the same as in Section 2.3.1. Figure 3e shows the result after the second search connects the structures remaining from Figure $3 d$.

\subsection{Texture Synthesis}

Once the surrounding structure has been completed through the damaged region, we can then fill in the damaged region with appropriate texture as constrained by the detected and completed structure. We use a pixel-based synthesis similar to that of Efros and Leung [13], with some modifications. As with their method, texture is propagated by estimating a conditional distribution of a pixel's value given its neighbors. This conditional distribution is computed by querying the sample image and finding all similar neighborhoods. Our method differs from [13] in three important ways:

1. Our method iterates more than once when determining which pixel to fill in and uses pixels filled in from the previous iteration in calculating the conditional distribution, similar to [15] (and in a way related to the loopy belief propagation in [20]).

2. Besides just referencing the neighboring pixels in the search, a smoothness prior based on the local color and intensity is also used.

3. Instead of searching through the entire image to build the conditional distribution, only the regions that are considered valid source data (as specified by the user) are used to influence the distribution.

While calculating these conditional distributions can be computationally expensive for large regions, and better or faster methods have been proposed $[1,8,12,15,22,23$, for example], we have found this method sufficient for this application, especially when the user has already limited the area for source textures. Many of these other techniques are more efficient for generating large amounts of texture because they use patches $[8,12]$ or build more efficient structures for comparing potentially matching pixels [22], but we found that the initial set-up required in these methods often negates these advantages when generating only small amounts of texture from limited source areas.

When searching for matching pixel areas, we consider only source pixels that share the same relationships relative to the detected and completed structures $[14,20]$. For example, in Figure $3 f$ we consider only the region marked by " $\mathrm{A}$ " when filling in the region marked with a "?". In some cases, such as in Figure 3g, there may still be some areas for which there are no sources areas that are topologically consistent with the structure. This situation is where the ambiguity in 


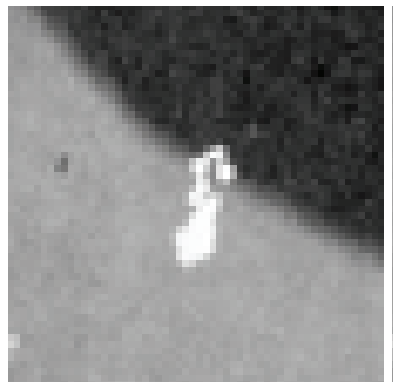

(a) Before

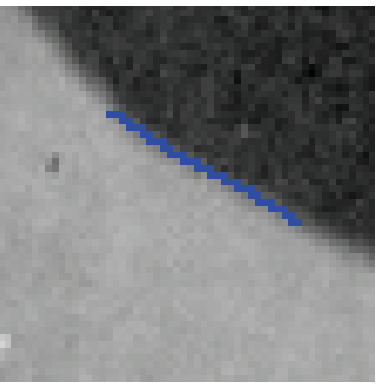

(b) Structure

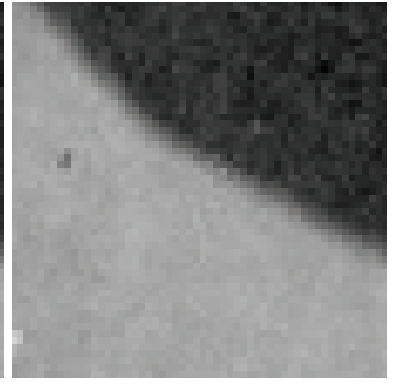

(c) After

Figure 4. Simple structure example. The scratch (a) is removed with a single mouse click. The algorithm preserves the edge between the textured dark and light regions by first detecting the structure (b) of the edges on each side of the damaged area, then filling in the damaged area with appropriate texture from each side of the edge (c).

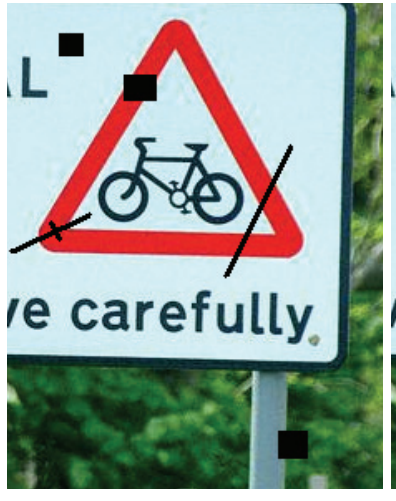

(a) Before

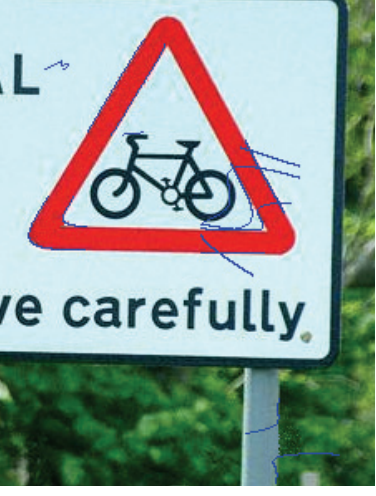

(b) Structure

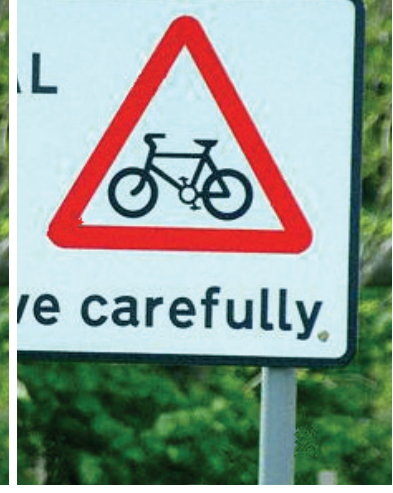

(c) After

Figure 5. Curved structure completion. Note that the curve in the lower left hand corner of the triangle is correctly completed, a difficult task for texture synthesis alone.

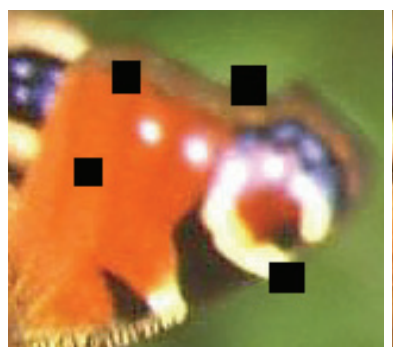

(a) Before

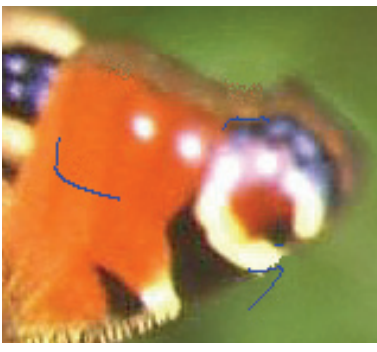

(b) Structure

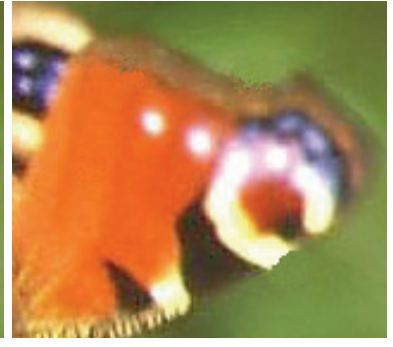

(c) After

Figure 6. Another example. A spurious edge was found in the middle of the butterfly's wing, which does not affect the result since the texture is the same on both sides. No structure was found in the soft edge along the top of the wing, but in this case failure to detect structure does not stop the algorithm from completing using texture alone. 


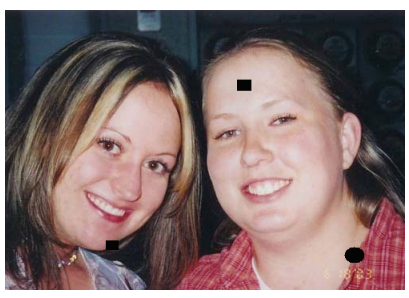

(a) Before

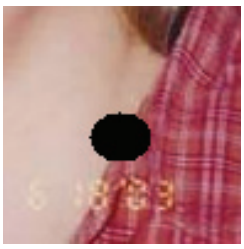

(c) Before (Inset)

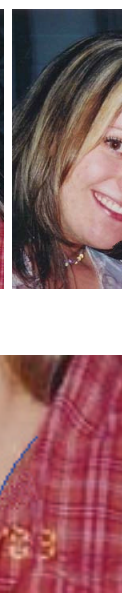

(d) Structure (Inset)

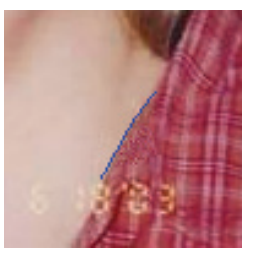

(b) After

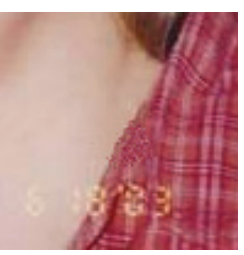

(e) After (Inset)
Figure 7. Structure and texture example. The two black squares and the black oval (a) are removed (b). One black square is located in the girl's forehead while the other black square is located between the other girl's chin and the background. The black oval occludes the edge between the red shirt and the girl's neck (c). The algorithm successfully fills in both structure (d) and texture (e).

filling-in occurs. In these cases, we must loosen the constraints and consider only some of the detected/completed structures but not all. If the user has directed us to fill in a certain way by gesturing with the mouse in the direction of the desired completion, we choose the pair of structure curves that are nearest in the direction orthogonal to the direction of the user's gesture, thus allowing us to fill in as directed by the user (Figure $3 \mathrm{~h}$ ).

Iterating the texture synthesis process not only produces better texture results, but it also causes multiple stochastically generated results to be presented to the user. Thus, the user can survey multiple options and indicate satisfaction with the results by clicking once more.

\section{Results and Discussion}

The interactive filling-in algorithm successfully detects and completes a variety of structure, including both curves and lines. In these examples, it should be kept in mind that the point is not to demonstrate that this method produces better images than previous methods. Rather, the point is to show that this framework for interactive repair can produce acceptable results in real time with minimal user interaction.

Figure 4 shows a simple example of structure completion. Notice that the detected and completed structure (blue

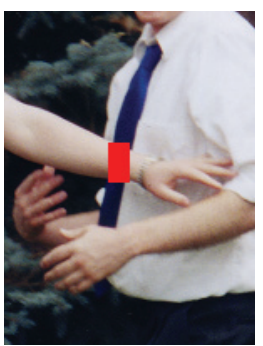

(a) Inset

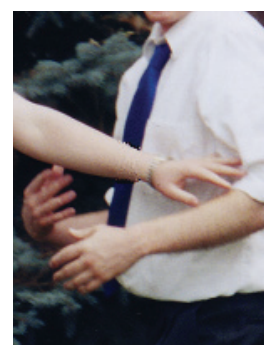

(b) Arm

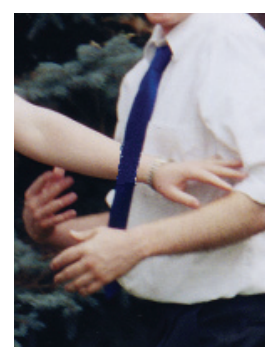

(c) Tie
Figure 8. Ambiguous case. The damaged region (a) may be completed with the arm over the tie (b) or the tie over the arm (c). Structure and texture are completed based on user motion indicating valid regions to search and desired structures to complete.

line) in Figure $4 \mathrm{~b}$ divides the edge between the textured dark and light regions. Figure $4 \mathrm{c}$ shows that this edge is preserved to correctly fill in the defect.

The interactive filling-in algorithm can also preserve curved structures. Figure 5 presents an example where the algorithm completes curves. The black lines and squares are successfully removed from the image, specifically the lines that occluded the lower left corner of the red triangle. Figure $5 \mathrm{~b}$ shows the curves that are completed in the defective region in the lower right corner of the red triangle. Notice some of these curves extrapolate beyond the valid edge point data, but they do not prevent a valid completion of the damaged portion (Figure 5c).

Figure 6 shows an example of the completion algorithm successfully filling in the damaged area in spite of spurious or missing edges.

In Figure 7a, the two black squares and the black oval are removed. Notice that the algorithm correctly fills in the texture of the faces and the texture of the red shirt. The images in Figure 7c-e show the detection and completion of the edge between the red shirt and the girl's neck.

Figures 1 and 8 show images with ambiguous structure. In Figure 8, the red square is removed. Structure and texture is completed based on the user motion in identifying which valid regions to search and which structures to complete. To complete the arm over the tie, the user moves the cursor along the arm when identifying which valid data to use to fill in. The user also strokes horizontally to complete the structure across the arm (Figure 8c). Likewise, if the user wants to complete the tie over the arm, they move the cursor along the tie to identify the blue tie structure and texture as the valid data to search to fill in. In addition, the user strokes vertically to complete the tie structure (Figure 8d). 


\section{Conclusion}

This paper has presented a framework for interactive image repair that allows a user to repair defects of moderate size through minimal interaction. In some cases the user need only click within the damaged area and loosely paint both the damaged area and the surrounding "good" image data. If necessary, the user can also guide the structurecompletion process through minimal mouse strokes, allowing proper reconstruction even in ambiguous cases.

This framework uses a combination of region growing (to detect the defect), edge detection (to detect the surrounding structure), curve fitting (to fit the surrounding structure and complete it through the defect), and constrained texture synthesis (to reconstruct the damaged area). While more sophisticated methods may be used for each of these portions of the framework, the overall process is limited by the need to maintain interactive speeds on current processors.

The method here is limited to fairly simple structures that extend across the defect in relatively straightforward ways, much like in [14]. If more complicated structure exists, one could use approaches similar to [20], in which the user explicitly draws the desired structure within the damaged region. We see these methods as complementary: minimal interaction for simplistic (but potentially ambiguous) structures, and more detailed interaction for more complicated structures.

\section{References}

[1] M. Ashikhmin. Synthesizing natural textures. In International Conference on Computer Graphics and Interactive Techniques (SIGGRAPH 2001), pages 217-226, 2001.

[2] C. Ballester, M. Bertalmio, V. Caselles, G. Sapiro, and J. Verdera. Filling-in by joint interpolation of vector fields and grey levels. IEEE Transactions on Image Processing, 10:1200-1211, August 2001.

[3] C. Ballester, V. Casalles, J. Verdera, M. Bertalmio, and G. Sapiro. A variational model for filling-in gray level and color images. In Proceedings International Conference on Computer Vision (ICCV), 2001.

[4] M. Bertalmio, A. L. Bertozzi, and G. Sapiro. Navier-stokes, fluid dynamics, and image and video inpainting. In Proceedings IEEE Computer Vision and Pattern Recognition (CVPR), 2001.

[5] M. Bertalmio, G. Sapiro, C. Ballester, and V. Caselles. Image inpainting. In Computer Graphics Proceedings, Annual Conference Series. ACM SIGGRAPH, 2000.

[6] M. Bertalmio, L. Vese, G. Sapiro, and S. Osher. Simultaneous structure and texture inpainting. In Computer Graphics Proceedings, Annual Conference Series, 2003.

[7] T. Chan and J. Shen. Non-texture inpainting by curvaturedriven diffusions (CCD). Technical Report 00-11, UCLA CAM, 2000.
[8] M. Cohen, J. Shade, S. Hiller, and O. Deussen. Wang tiles for image and texture generation. In International Conference on Computer Graphics and Interactive Techniques (SIGGRAPH 2003), volume 22, pages 287-294, July 2003.

[9] A. Criminisi, P. Pérez, and K. Toyama. Object removal by exemplar-based inpainting. In Proceedings IEEE Conference on Computer Vision and Pattern Recognition, volume 2, pages 721-728, June 2003.

[10] L. Demanet, B. Song, and T. Chan. Image inpainting by correspondance maps: A deterministic approach. Technical Report 03-40, UCLA CAM, August 2003.

[11] I. Drori, D. Cohen-Or, and H. Yeshurun. Fragment-Based image completion. In International Conference on Computer Graphics and Interactive Techniques (SIGGRAPH 2003), pages 303 - 317, July 2003.

[12] A. Efros and W. Freeman. Image quilting for texture synthesis and transfer. In International Conference on Computer Graphics and Interactive Techniques (SIGGRAPH 2001), August 2001.

[13] A. Efros and T. Leung. Texture synthesis by non-parametric sampling. In IEEE International Conference on Computer Vision, pages 1033-1038, September 1999.

[14] J. Jia and C. Tang. Image repairing: robust image synthesis by adaptive ND tensor voting. In Proceedings IEEE Conference on Computer Vision and Pattern Recognition, volume 1, pages 643-650, June 2003.

[15] V. Kwatra, I. Essa, A. Bobick, and N. Kwatra. Texture optimization for example-based synthesis. In ACM SIGGRAPH 2005, 2005.

[16] Y. Ohta, T. Kanade, and T. Sakai. Color information for region segmentation. Computer Graphics and Image Processing, 13:222-241, July 1980.

[17] M. Oliveira, B. Bowen, R. McKenna, and Y. Chang. Fast digital image inpainting. In International Conference on $\mathrm{Vi}$ sualization, Image and Image Processing, pages 261-266, September 2001.

[18] P. Perona and J. Malik. Scale-space and edge detection using anisotropic diffusion. IEEE Transactions on Pattern Analysis and Machine Intelligence, 12:629-639, 1990.

[19] T. W. Sederberg, F. Chen, and K. Klimaszewski. Rotated explicit curves. In Proceedings of the Fifth International Conference on CAD \& Graphics, pages 358-361, 1997.

[20] J. Sun, L. Yuan, J. Jia, and H.-Y. Shum. Image completion with structure propagation. In Proceedings of ACM SIGGRAPH 2005, pages 861-868, 2005.

[21] A. Telea. An image inpainting technique based on the fast marching method. Journal of Graphics Tools, 9(1):25-36, 2004.

[22] L. Wei and M. Levoy. Fast texture synthesis using treestructured vector quantization. In International Conference on Computer Graphics and Interactive Techniques (SIGGRAPH 2000), pages 479-488, July 2000.

[23] S. Zelinka and M. Garland. Towards real-time texture synthesis with the jump map. In Proceedings Eurographics 2002, 2002. 